European Journal of Human Genetics (2009) 17, 284-286

(C) 2009 Macmillan Publishers Limited All rights reserved 1018-4813/09 \$32.00

www.nature.com/ejhg

\section{Does epidermal thickening explain GJB2 high carrier frequency and heterozygote advantage?}

European Journal of Human Genetics (2009) 17, 284-286; doi:10.1038/ejhg.2008.225; published online 3 December 2008

In the post-human-genome area, the challenge is to derive details of heritable variation in relation to how human variation reflects adaptation to the different environments. Heterozygote advantage represents a superior genetic adaptation presumably explaining the presence of the allele at frequencies above those expected from a simple replacement of a homozygous lethal allele by mutation alone. A successful adaptation requires natural selection acting on that part of the body that makes a difference in survival. There is ongoing discussion as to what extent inherited diseases may be phenotypically balanced by a selective heterozygous advantage. We know for sure that sickle cell anemia and some other red blood cell disorders are selected for by malaria, ${ }^{1}$ whereas a selective carrier advantage for cystic fibrosis has been recently shown. ${ }^{2}$ For other common inherited diseases counterbalancing mechanisms have been only hypothesized. Congenital hearing loss accounts for about 1 in 1000 infants and approximately $80 \%$ of the cases are inherited as an autosomal recessive trait. Connexin 26 (GJB2) gene, a component of a gap junction, is a major gene for these forms, and a large proportion of recessive cases are due to mutations in this gene. ${ }^{3}$ Different common populationspecific mutations have been so far described. ${ }^{4,5}$ Among them, mutation $35 \mathrm{delG}$ explains up to $70 \%$ of the alleles detected in Caucasians, with carrier frequencies ranging from $1 / 30$ in the Mediterranean area to $1 / 70$ in northern Europe. ${ }^{5}$ The high carrier frequency of the GJB2 mutations in many ethnic groups strongly suggests that there may be a heterozygous advantage. Why GJB2 carriers are so frequent? Quite recently, it has been reported that carriers of R134W allele, present in some African countries such as Ghana, ${ }^{6,7}$ show a thicker epidermis than wild-type ones. ${ }^{8}$ Moreover, in vitro studies have further supported this finding showing that cells expressing the R134W allele: (a) form a significantly thicker epidermis in an organotypic co-culture skin model, (b) show an increased migration, (c) are significantly less susceptible to cellular invasion by the enteric pathogen Shigella flexneri than wild-type cells. ${ }^{9}$ Another study has shown that the transfection of some deafness-associated mutant constructs resulted in a statistically significant reduction in cell death when compared with wild-type ones. ${ }^{10}$ Thus, researchers hypothesized that an increased cell survival may explain a thicker epidermis due to an extended terminal differentiation program leading to an improved barrier against infection. Moreover, some specific GJB2 mutated alleles lead to skin diseases in which abnormal thickening of the skin is present. ${ }^{11}$ If epidermal thickening is the phenomenon underlying heterozygote advantage, should we be able to find an increased thickness of the epidermis in GJB2 carriers? To answer this question and possibly to explain the increased $35 \mathrm{delG}$ carrier frequency, we have developed an accurate sonographic protocol to measure epidermal thickness able to discriminate between normal controls and GJB2 carriers. ${ }^{12}$ Table 1 report the data on epidermal thickness in a series of 240 Italian normal controls (134 females and 106 males) aged from 20 to 86 years. They have been then compared with those obtained in a series of fifty-four 35delG obligate carriers (29 females and 25 males). Results of the analysis are shown in Figure 1, in which two regression lines are calculated for each of the two groups (normal controls and carriers). A clear tendency to thickening along with age is present in both sexes $(P<2.0 \mathrm{e}-16)$, being more relevant in males. Two clusters are easily distinguishable, one defined by controls and one by carriers. An ANCOVA analysis showed that three variables (GJB2 status, age and gender) explain up to $75 \%$ of epidermal thickness variability $(P<2.1 \mathrm{e}-16)$. Removing the $G J B 2$ status, the impact of the remaining two variables (ie, gender and age) is of only $26 \%$. The difference between controls and GJB2 carriers cannot be explained with any social or occupational reason (ie, lifestyle, job and so on).

The basic mechanism underlying epidermal thickening in 35delG carriers (loss of function mutants) is still

Table 1 Epidermal thickness in normal controls and GJB2 carriers

\begin{tabular}{|c|c|c|c|c|c|c|c|c|}
\hline & \multicolumn{4}{|c|}{ Normal controls } & \multicolumn{4}{|c|}{ Carriers } \\
\hline & Mean & Min & Max & $S D \pm$ & Mean & Min & Max & $S D \pm$ \\
\hline Females & 0.38 & 0.17 & 0.72 & 0.11 & 0.81 & 0.59 & 1.16 & 0.13 \\
\hline Males & 0.41 & 0.18 & 0.88 & 0.14 & 0.93 & 0.53 & 1.14 & 0.16 \\
\hline
\end{tabular}

Data (in $\mathrm{mm}$ ) on mean thickness, minimum and maximum as well as standard deviations divided by sex are reported either for normal controls and $35 \mathrm{delG}$ carriers. 

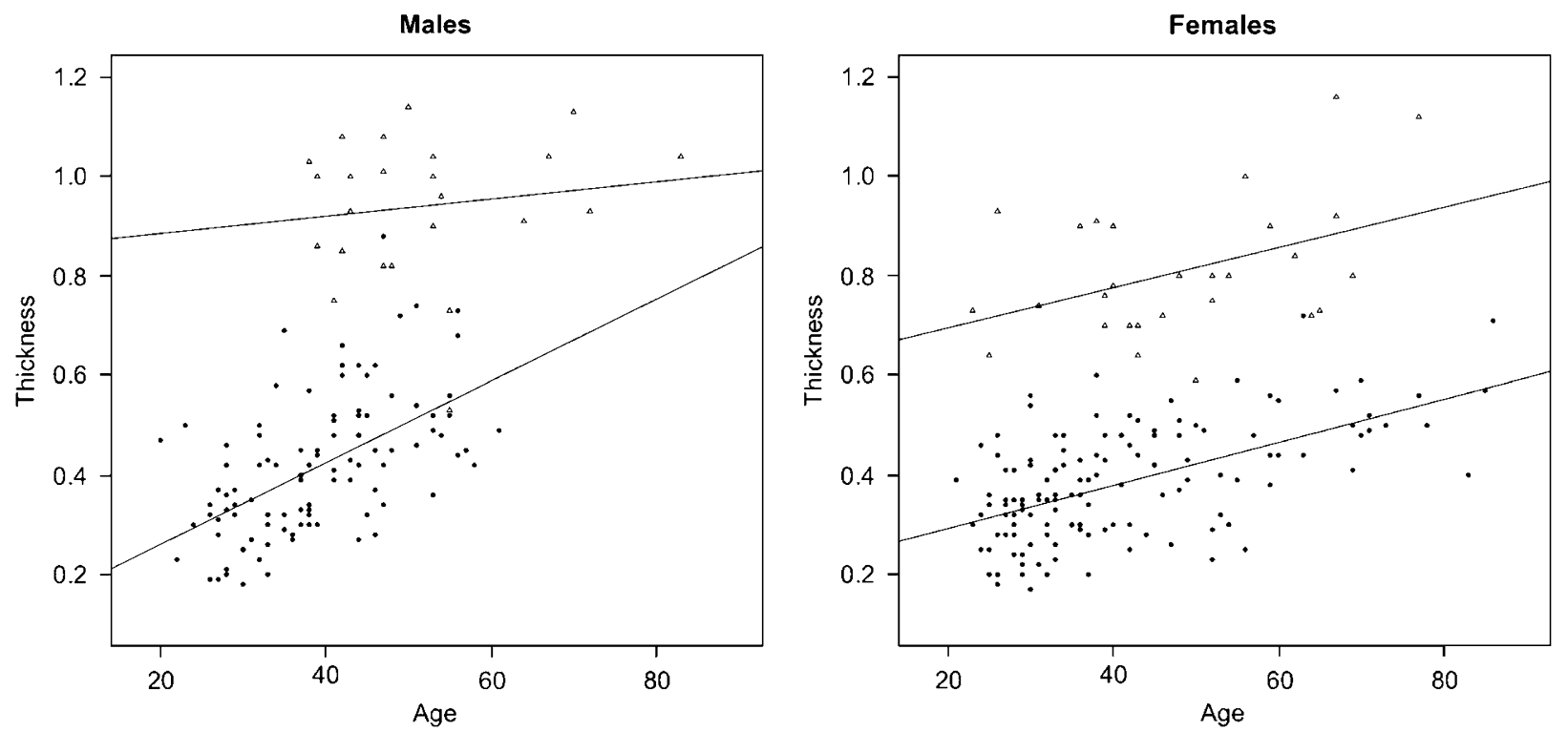

Figure 1 Regression analysis of epidermal thickness in a series of normal controls and 35delG GJB2 carriers. P-values have been obtained using a regression analysis of epidermal thickness using status, age and sex as covariates. Statistical analysis and graphs have been carried out using R software version 2.7.0. Triangles: carriers; Black dots: normal controls. Measurements of epidermal thickness have been obtained using a ultrasound linear large band probe ranging from 6 to $15 \mathrm{MHz}$ with the probe placed at the midline of the forehead.

unknown. Might we speculate a possible underlying mechanism? Keratinocytes are the prevalent cell type of the epidermis, the multilayered cornified epithelium that provides the cellular basis of the outermost barrier between each subject and his/her environment. By this barrier function the epidermis protects against a variety of environmental hazards such as dehydration and mechanical stress. The sophisticated cooperation between soluble growth factors, cell-matrix interactions and cell-to-cell communications are major parts of the machinery regulating keratinocyte migration. Loosening, at least in part, gap junction structure might disturb cell-to-cell interaction, leading to keratotic changes and epidermal thickening, thus, at the end, to a stronger mechanical barrier.

Is the presence of epidermal thickening enough to explain the carrier advantage or other more subtle functional advantages could also be gained in other tissues? The bacterium Shigella flexneri, a human intestinal pathogen, causing dysentery by invading the epithelium of the colon and responsible, worldwide, for an estimated 165 million episodes of shigellosis and 1.5 million deaths per year, has been shown to induce the opening of Connexin 26 hemichannels providing evidence that pathogen-induced opening of Connexin 26 may promote bacterial invasion. ${ }^{13}$ Moreover, as already mentioned, in vitro studies have shown that cells expressing the R134W allele are significantly less susceptible to cellular invasion by the enteric pathogen Shigella flexneri than wild-type cells. ${ }^{9}$ Taking into account all these findings, might we hypothesize a GJB2 carrier advantage similar to that observed with cystic fibrosis, in which CFTR mutants are restricting the invasion of Salmonella typhi into epithelial cells and thus are providing protection against typhoid fever? ${ }^{14}$

In conclusion, although the presence of epidermal thickening in GJB2 carriers is now almost proven, additional studies are needed to: (a) assess if this mechanism does indeed reduce bacterial invasion and infection rates through the skin, (b) better understand if this mechanism could also apply to other tissues such as intestinal epithelia.

Pio D'Adamo ${ }^{1}$, Veronica Ileana Guerci ${ }^{2}$, Antonella Fabretto $^{2}$, Flavio Faletra ${ }^{2}$, Domenico Leonardo Grasso ${ }^{3}$, Luca Ronfani ${ }^{4}$, Marcella Montico ${ }^{4}$, Marcello Morgutti ${ }^{1}$, PierPaolo Guastalla ${ }^{5}$ and Paolo Gasparini ${ }^{\star, 1,2}$

${ }^{1}$ Medical Genetics, Department of Laboratory Medicine, Institute of Child Health IRCCS-Burlo, Trieste, Italy;

${ }^{2}$ Medical Genetics, Department of Reproductive Sciences and Development, University of Trieste, Trieste, Italy;

${ }^{3}$ Audiology Unit, Institute of Child Health IRCCS-Burlo Garofolo, Trieste, Italy; ${ }^{4}$ Epidemiology and Biostatistics, Institute of Child Health IRCCS-Burlo Garofolo, Trieste, Italy;

${ }^{5}$ Radiology Unit, Institute of Child Health, IRCCS-Burlo Garofolo, Trieste, Italy

*Correspondence: Dr P Gasparini, Medical Genetics, Department of Reproductive Sciences and Development, Institute of Child Health IRCCS-Burlo Garofolo, University of Trieste, Via dell'Istria 65, Trieste 34141, Italy. Tel: +39040 378 5275; Fax: + 39040378 5540; E-mail: gasparini@burlo.trieste.it 


\section{References}

1 Roth Jr EF, Raventos-Suarez C, Rinaldi A, Nagel RL: Glucose-6phosphate dehydrogenase deficiency inhibits in vitro growth of Plasmodium falciparum. Proc Natl Acad Sci USA 1983; 80: 298-299.

2 Gabriel SE, Brigman KN, Koller BH et al: Cystic fibrosis heterozygote resistance to cholera toxin in the cystic fibrosis mouse model. Science 1994; 266: 107-109.

3 Zelante L, Gasparini P, Estivill X et al: Connexin26 mutations associated with the most common form of non-syndromic neurosensory autosomal recessive deafness (DFNB1) in Mediterraneans. Hum Mol Genet 1997; 6: 1605-1609.

4 Lucotte G, Dieterlen F: The 35delG mutation in the connexin 26 gene (GJB2) associated with congenital deafness: European carrier frequencies and evidence for its origin in ancient Greece. Genet Test 2005; 9: 20-25.

5 Gasparini P, Rabionet R, Barbujani G et al: High carrier frequency of the $35 \mathrm{delG}$ deafness mutation in European populations. Genetic Analysis Consortium of GJB2 35delG. Eur J Hum Genet 2000; 8: 19-23.

6 Brobby GW, Müller-Myhsok B, Horstmann RD: Connexin 26 R143W mutation associated with recessive nonsyndromic sensorineural deafness in Africa. N Engl J Med 1998; 338: 548-550.

7 Hamelmann C, Amedofu GK, Albrecht $\mathrm{K}$ et al: Pattern of connexin 26 (GJB2) mutations causing sensorineural hearing impairment in Ghana. Hum Mutat 2001; 18: 84-85.

8 Meyer CG, Amedofu GK, Brandner JM et al: Selection for deafness? Nat Med 2002; 8: 1332-1333.

9 Man YK, Trolove C, Tattersall D et al: A deafness-associated mutant human connexin 26 improves the epithelial barrier in vitro. J Membr Biol 2007; 218: 29-37.

10 Common JE, Di W-L, Davies D et al: Further evidence for heterozygote advantage of GJB2 deafness mutations: a link with cell survival. J Med Genet 2004; 41: 573-575.

11 Lai-Cheong JE, Arita K, McGrath JA: Genetic diseases of junctions. J Invest Dermatol 2007; 127: 2713-2725.

12 Guastalla P, Guerci V, Faretto A et al: Epidermal sonography can detect epidermal thickening in GJB2 carriers. Radiology, (In press).

13 Tran Van Nhieu G, Clair C, Bruzzone R et al: Connexindependent inter-cellular communication increases invasion and dissemination of Shigella in epithelial cells. Nat Cell Biol 2003; 5: $720-726$.

14 Pier GB, Grout M, Zaidi T et al: Salmonella typhi uses CFTR to enter intestinal epithelial cells. Nature 1998; 393: 79-82. 